\title{
ON MEAN-PERIODICITY. II
}

\author{
BY \\ EDWIN J. AKUTOWICZ( $\left.{ }^{(}\right)$
}

\begin{abstract}
This paper is devoted to the problem of representing all solutions of certain homogeneous convolution equations through series of exponential polynomials. This representation is sought in the dual space $\mathscr{M}^{\prime}$ of a function space $\mathscr{M}$, the latter consisting of entire functions satisfying growth conditions in horizontal directions. The space $\mathscr{M}$ is a Fréchet space, which fact permits a simpler and more thorough treatment than that given in the paper [1]. The technique used here is based upon a method developed by L. Ehrenpreis [5] and V. P. Palamodov [3] in the theory of differential equations with constant coefficients. We map the Fourier transform space $\mathscr{F} \mathscr{M}$ into a space of sequences,
\end{abstract}

$$
\rho: \mathscr{F} \mathscr{M} \ni F \rightarrow\left(F\left(\lambda_{1}\right), F^{\prime}\left(\lambda_{1}\right), \ldots, F^{\left(p_{1}-1\right)}\left(\lambda_{1}\right), F\left(\lambda_{2}\right), \ldots, F^{\left(p_{2}-1\right)}\left(\lambda_{2}\right), \ldots\right),
$$

where $\left\{\lambda_{k}\right\}$ is the spectrum with multiplicity of a mean-periodic element of the dual space $\mathscr{M}^{\prime}$. The crucial point is to identify the quotient space $\mathscr{F} \mathscr{M} /$ ker $\rho$.

In a recent paper [1] we have studied mean-periodic distributions that act in a certain fundamental space $M$ of holomorphic functions. The principal result of the first chapter of that paper was the spectral analysis and synthesis of such distributions; in fact, it was shown that the formal series of exponentials associated with a mean-periodic element belonging to the dual space $M^{\prime}$ of $M$ is uniformly convergent on each set of a reasonable class of bounded sets of the fundamental space $M$.

The present paper, which is entirely new and independent of [1], deals with a closely related problem in a simpler way. The simplification arises by taking advantage of a natural extension of a method used by V. P. Palamodov in the theory of differential equations with constant coefficients (cf. [3], which contains ample bibliographical and historical references) and by operating in a fundamental space $\mathscr{M}$ of simpler structure than the space $M$ of [1]. The present space $\mathscr{M}$ is a Fréchet space (a projective limit of Banach spaces), whereas $M$ was a mixed projectiveinductive limit.

Our goal in the following pages is to give an affirmative and explicit solution of the spectral analysis and synthesis problem for mean-periodic distributions of $\mathscr{M}^{\prime}$.

Received by the editors March 31, 1970.

AMS 1970 subject classifications. Primary 46F15, 46E20; Secondary 42A96, 30A.80.

Key words and phrases. Spectral analysis and synthesis, mean-periodic distributions, homogeneous convolution equations, linear topological space of entire functions and its dual, Fourier transform, Fantappiè indicator, interpolation.

( ${ }^{1}$ Supported in part by Air Force Office of Scientific Research grant AF-AFOSR-69-1712 and by European Research Office, Frankfurt. 
The fundamental space $\mathscr{M}$. Let us fix a positive number $a$ once and for all. We shall systematically denote the Fourier transform of an integrable function defined on the real line $\boldsymbol{R}$ by the corresponding capital letter; thus

$$
\mathscr{F}_{\varphi}(x)=\Phi(x)=\int_{R} \varphi(t) e^{i x t} d t
$$

Let $\mathscr{M}$ denote the vector space of all entire functions $m$ such that the corresponding Fourier transforms $M$ are holomorphic in the horizontal strip

$$
A=\{z=x+i y:|y|<a\}
$$

and satisfy

$$
\|M\|_{k n} \stackrel{\text { def }}{\equiv} \sup _{|y| \leqq a-1 / n}\left\|M(x+i y) e^{k|x|}\right\|<\infty
$$

for all positive integers $k$ and $n$. The norm appearing in the right-hand member of (1) is that of the classical Lebesgue space $L^{2}$ over the real line $\boldsymbol{R}$.

We put $\|m\|_{k n}=\|M\|_{k n}, \forall k, n$, in order to obtain a Fréchet space $\mathscr{M}$.

Let us remark at once that the space $\mathscr{M}$ is invariant under real translations. Indeed, if $\varphi \in \mathscr{M}$ and $\varphi_{\xi}(t)=\varphi(t-\xi), \xi$ real, we have

$$
\mathscr{F}\left(\varphi_{\xi}\right)(x+i y)=e^{i(x+i y) \xi} \mathscr{F} \varphi(x+i y)
$$

and

$$
\sup _{|y| \leqq a-1 / n}\left\|\mathscr{F}\left(\varphi_{\xi}\right)(x+i y) e^{k|x|}\right\| \leqq e^{a \xi} \sup _{|y| \leqq a-1 / n}\left\|\mathscr{F} \varphi(x+i y) e^{k|x|}\right\|<\infty .
$$

It is then clear that the dual space $\mathscr{M}^{\prime}$ also admits real translations. We can therefore define an element $\lambda \in \mathscr{M}^{\prime}$ to be mean-periodic in $\mathscr{M}^{\prime}$ if the closed linear span of the set of translates $\left\{\lambda_{\xi}: \xi \in R\right\}$ is strictly contained in $\mathscr{M}^{\prime}$. Here, on account of convexity, it makes no difference whether we take the weakly or strongly closed linear span.

It is also clear that $\mathscr{M}$ is an algebra under convolution on $\boldsymbol{R}$.

We aim to prove the following theorem concerning the space $\mathscr{M}$, which characterises the mean-periodic distributions in $\mathscr{M}^{\prime}$ through their harmonic analysis.

THEOREM. Each mean-periodic element $\lambda$ in the dual space $\mathscr{M}^{\prime}$ is the sum of a weakly convergent series

$$
\lambda=\sum_{k} \sum_{r=0}^{p_{k}-1} c_{r k} x^{r} \exp \left(i \lambda_{k} x\right),
$$

where $\left\{\lambda_{k}\right\}_{k=1,2, \ldots}$ is a sequence of complex numbers (the spectrum of $\lambda$ ) contained in a horizontal strip $\left\{|y|<a^{\prime}\right\}, a^{\prime}<a$, strictly contained in $A$ and $\left\{p_{k}\right\}$ is a sequence of positive integers ( $p_{k}$ is the multiplicity of $\lambda_{k}$ ). The sequence $\left\{\lambda_{k}\right\}$ coincides with the set of zeros of a function $M_{0} \in \mathscr{F} \mathscr{M}$ which vanishes at $\lambda_{k}$ with multiplicity $p_{k}$. The coefficient sequence $\left\{c_{r k}\right\}$ satisfies a growth condition

$$
\sum_{r, k}\left|c_{r k}\right| W_{q r k}^{-1} \exp \left(-q\left|\operatorname{Re} \lambda_{k}\right|\right)<\infty, \quad \exists q<\infty,
$$


where $\left\{W_{q r k}\right\}$ is an explicitly determined sequence depending only upon the number $q$ and upon the sequence $\left\{\lambda_{k}\right\}$. The three sequences $\left\{\lambda_{k}\right\},\left\{p_{k}\right\},\left\{c_{r k}\right\}$ are uniquely determined by $\lambda$.

Conversely, if $\lambda \in \mathscr{M}^{\prime}$ is the sum of a weakly convergent series (2) such that the growth condition (3) is satisfied, and if there is a function $M_{0} \in \mathscr{F} \mathscr{M}$ which vanishes at $\lambda_{k}$ with multiplicity $p_{k}$, then $\lambda$ is mean-periodic in $\mathscr{M}^{\prime}$.

A series of lemmas. An essential part of the proof is contained in the following lemmas, of which the most delicate is Lemma 3.

Lemma 1. The closed linear span $\mathscr{L}$ of the translates of an element $m \in \mathscr{M}$ is an ideal under convolution; that is, $m * n \in \mathscr{L}$ for every $n \in \mathscr{M}$.

Proof. With obvious notations, we have $(z=x+i y)$

$$
\begin{aligned}
\| \sum_{j} m\left(t-\xi_{j}^{\prime}\right) & n\left(\xi_{j}^{\prime}\right) \Delta \xi_{j}^{\prime}-\sum m\left(t-\xi_{j}^{\prime \prime}\right) n\left(\xi_{j}^{\prime \prime}\right) \Delta \xi_{j}^{\prime \prime} \|_{k n} \\
& =\sup _{|y| \leqq a-1 / n}\left\|M(z)\left(\sum \exp \left(i z \xi_{j}^{\prime}\right) n\left(\xi_{j}^{\prime}\right) \Delta \xi_{j}^{\prime}-\sum \exp \left(i z \xi_{j}^{\prime \prime}\right) n\left(\xi_{j}^{\prime \prime}\right) \Delta \xi_{j}^{\prime \prime}\right) e^{k|x|}\right\|_{L^{2}} \\
& \leqq \sup _{|y| \leqq a-1 / n}\left|\sum \exp \left(i z \xi_{j}^{\prime}\right) n\left(\xi_{j}^{\prime}\right) \Delta \xi_{j}^{\prime}-\sum \exp \left(i z \xi_{j}^{\prime \prime}\right) n\left(\xi_{j}^{\prime \prime}\right) \Delta \xi_{j}^{\prime \prime}\right| \cdot\|m\|_{k n},
\end{aligned}
$$

a quantity which tends to zero for each $k, n$. Recalling that $\mathscr{M}$ is complete, the result follows.

We shall require an integral representation of the Fourier transform $\Lambda=\mathscr{F} \lambda$ through what is essentially the Fantappiè indicator. Formally, this is just the "Cauchy integral"

$$
z \rightarrow\langle\Lambda, 1 /(z-t)\rangle
$$

but it should be realised that the Cauchy kernel $t \rightarrow 1 /(z-t)$ does not belong to the space $\mathscr{F} \mathscr{M}$ in which $\Lambda$ acts. We shall need (4) later on even for some points $z$ situated inside the strip $A$. Hence some attention has to be given to extending $\Lambda$ properly, so that (4) will make sense.

Since $\Lambda$ is continuous in the Fréchet topology given by the family of norms (1), $\Lambda$ is bounded on some neighborhood of $0,\|M\|_{k n} \leqq 1, \exists k, n$. By the Cauchy integral formula, we can suppose $k$ and $n$ to be such that $\Lambda$ is bounded on a ball

$$
\|M\|_{k n}=\operatorname{sef}_{|\operatorname{Im} z| \leqq a-1 / n}\left|M(z) e^{k|\operatorname{Re} z|}\right| \leqq 1, \quad M \in \mathscr{F} \mathscr{M} .
$$

Let $B_{k n}$ denote the Banach space of all functions $F$ continuous for $|y| \leqq a-1 / n$, holomorphic for $|y|<a-1 / n$, such that $\|F\|_{k n}<\infty$. Then $\Lambda$ can be extended to a continuous linear form on $B_{k n}$. We fix such an extension, denoted again by $\Lambda$.

Let $M_{0}$ be a function of $\mathscr{F} \mathscr{M}$ and consider the factorisation

$$
M_{0}=B_{0} M_{1},
$$


where $B_{0}$ is the Blaschke product relative to any horizontal strip $H_{v} \supset H_{n}(\nu>n)$ over the zeros of $M_{0}$ lying in $H_{\nu}\left({ }^{2}\right)$. We can suppose that $|B| \equiv 1$ on the boundary $\partial H_{v}$. Since $M_{1}$ has no zeros in $\bar{H}_{v}$, let

$$
M_{2}=\sqrt{ } M_{1} \text { on } \bar{H}_{v} .
$$

Then

$$
\left\|M_{0} / M_{2}\right\|_{k n}<\infty .
$$

Define, using the extension of $\Lambda$ to the space $B_{k n}$,

$$
L(z)=\frac{1}{2 \pi i} \frac{\left\langle\Lambda, M_{2} /(z-t)\right\rangle}{M_{2}(z)}, \quad z \in H_{v} \mid \bar{H}_{n} .
$$

LEMMA 2. There exists a constant $C_{k n}$ such that the inequality

$$
\left|M_{0}(z) L(z)\right| \leqq C_{k n}\left|M_{2}(z)\right|
$$

holds if $z \in H_{\nu} \mid \bar{H}_{n}$ and dist $\left(z, H_{n}\right) \geqq \alpha>0$.

Proof. By definition, $M_{2}(z) L(z)=\left\langle\Lambda, M_{2} /(z-t)\right\rangle$, where $z$ runs over the two horizontal strips $H_{v} \mid \bar{H}_{n}$ near the boundary of $A$, and where $M_{2}$ is related to $M_{0}$ through (6) and (7). Since $M_{2} \in B_{k n}$ so that $t \rightarrow M_{2}(t) /(z-t) \in B_{k n}\left(z \in H_{v} \mid \bar{H}_{n}\right)$ as well, and since $\Lambda$ is a continuous linear form on the Banach space $B_{k n}$, we obtain the bound

$$
\left|M_{2}(z) L(z)\right| \leqq\|\Lambda\| \cdot\left\|M_{1}(t) /(z-t)\right\|_{k n .} .
$$

Since dist $\left(z, H_{n}\right) \geqq \alpha>0$,

$$
\left\|M_{1}(t) /(z-t)\right\|_{k n} \leqq K\left\|M_{1}\right\|_{k n}
$$

for a constant $K=K_{\alpha}$. Therefore (10) and (11) yield $C_{k n}$ such that $\left|M_{2}(z) L(z)\right|$ $\leqq C_{k n}<\infty$. Thus, it follows that

$$
\left|M_{0}(z) L(z)\right|=\left|B_{0}(z) M_{2}(z) \cdot M_{2}(z) L(z)\right| \leqq C_{k n}\left|M_{2}(z)\right|
$$

for $z$ as stated.

Let the contour $\Gamma$ consist of two horizontal lines, one lying in the lower strip of $\left.H_{v}\right\rangle \bar{H}_{n}$ and directed to the right and one in the upper strip of $H_{v} \backslash \bar{H}_{n}$, directed to the left.

Under the above conditions, we have the following:

Lemma 3. The action of $\lambda \in \mathscr{M}^{\prime}$ on the elements of the principal ideal $m_{0} * \mathscr{M}$, $\mathscr{F} m_{0}=M_{0}$, is given by

$$
\left\langle\lambda, m_{0} * m\right\rangle=2 \pi\left\langle\Lambda, M_{0} M\right\rangle=\int_{\Gamma} L(z) M(z) M_{0}(z) d z .
$$

(2) $H_{n}=\{z=x+i y:|y|<a-1 / n\}$. 
Proof. The proof consists in justifying the following two equalities:

$$
\begin{aligned}
\frac{1}{2 \pi i} \int_{\Gamma} \frac{M_{0}(z) M(z)}{M_{2}(z)}\left\langle\Lambda, \frac{M_{2}}{z-t}\right\rangle d z & =\left\langle\Lambda, \frac{M_{2}}{2 \pi i} \int_{\Gamma} \frac{M_{0}(z) M(z)}{M_{2}(z)} \frac{d z}{z-t}\right\rangle \\
& =\left\langle\Lambda, M_{0} M\right\rangle .
\end{aligned}
$$

The second equality follows at once from the Cauchy integral representation

$$
\frac{M_{0}(t) M(t)}{M_{2}(t)}=\frac{1}{2 \pi i} \int_{\Gamma} \frac{M_{0}(z) M(z)}{M_{2}(z)} \frac{d z}{z-t}, \quad t \in \bar{H}_{n},
$$

which is certainly valid in view of (8).

As for the first equality, the integral on the left certainly exists, by Lemma 2 . On the other hand, the Riemann sums for the integral (12), multiplied by $M_{2}$, converge in the norm of $B_{k n}$ (cf. the proof of Lemma 1), and the first equality follows.

LEMMA 4. If $\left\langle\Lambda, M M_{0}\right\rangle=0$ for all $M \in \mathscr{F} \mathscr{M}$, then the product $L M_{0}$ has an analytic extension throughout the strip $H_{n}$.

Proof. Let $\zeta$ be a point outside of the strip $A$. Then for every $M \in \mathscr{F} \mathscr{M}$, the function $z \rightarrow M(z) /(z-\zeta), z \in A$, again belongs to $\mathscr{F} \mathscr{M}$. Therefore, according to the hypothesis and Lemma 3,

$$
\int_{\Gamma} \frac{L(z) M(z) M_{0}(z)}{z-\zeta} d z=0
$$

By continuity, (13) continues to hold for all points $\zeta$ outside the strip $H_{\Gamma}$ bounded by $\Gamma$. After a conformal mapping of $H_{\Gamma}$ we can replace (13) by a Cauchy integral over the unit circle which vanishes identically outside the unit circle. Therefore the numerator of the integrand must have an analytic extension to the interior of the unit circle. Mapping back, it follows that the product $L M M_{0}$ has an analytic extension over the strip $H_{\Gamma}$; since $M$ can be any function of $\mathscr{F} \mathscr{M}$, this implies that $L M_{0}$ has an analytic extension over $H_{\Gamma} \supset H_{n}$, as asserted.

Proof of the theorem. Let $\lambda$ denote any mean-periodic distribution in $\mathscr{M}^{\prime}$. Then there exists a nontrivial element $m_{0} \in \mathscr{M}$ such that $\left\langle\lambda,\left(m_{0}\right)_{\xi}\right\rangle=0, \forall \xi \in \boldsymbol{R}$. According to Lemma 1 , this implies that $\left\langle\lambda, m_{0} * m\right\rangle=0, \forall m \in \mathscr{M}$. Taking Fourier transforms, it follows that $\left\langle\Lambda, M_{0} M\right\rangle=0, \forall M \in \mathscr{F} \mathscr{M}$. Consider now the function $L$ defined by (9). According to Lemma 4, the product $M_{0} L$ can be regarded as a holomorphic function in a strip $H_{n}$. Therefore the singularities of $L$ are isolated poles $\left\{\lambda_{k}\right\}_{k=1,2, \ldots} \subset H_{n}$, cancelled by the zeros of $M_{0}$. Let $p_{k}$ denote the multiplicity of the pole of $L$ at $\lambda_{k}$.

Now take the mapping (cf. [3])

$$
\rho: \Psi \rightarrow\left(\Psi\left(\lambda_{1}\right), \Psi^{\prime}\left(\lambda_{1}\right), \ldots, \Psi^{\left(p_{1}-1\right)}\left(\lambda_{1}\right), \Psi\left(\lambda_{2}\right), \ldots, \Psi^{\left(p_{2}-1\right)}\left(\lambda_{2}\right), \ldots\right) .
$$

This maps the space $\mathscr{F} \mathscr{M}$ into a certain sequence space

$$
S=\left\{\sigma=\left\{\sigma_{r k}\right\}: r=0, \ldots, p_{k}-1, k=1,2, \ldots\right\} .
$$


For each $\Psi \in \mathscr{F} \mathscr{M}$, we have the representation

$$
\begin{aligned}
\Psi^{(r)}\left(\lambda_{k}\right) \exp \left( \pm q \lambda_{k}\right) & =\frac{1}{2 \pi i} \int_{\Gamma} \frac{\Psi^{(r)}(z) e^{ \pm q z}}{z-\lambda_{k}} d z \\
& =\frac{(-1)^{r}}{2 \pi i} \int_{\Gamma} \Psi(z) \frac{d^{r}}{d z^{r}}\left(\frac{e^{ \pm q z}}{z-\lambda}\right) d z \\
& =\frac{(-1)^{r}}{2 \pi i} \int_{\Gamma} \Psi(z) e^{ \pm q z} P_{r q}\left(\frac{1}{z-\lambda_{k}}\right) d z
\end{aligned}
$$

where $P_{r q}\left(1 /\left(z-\lambda_{k}\right)\right)$ is a polynomial of degree $r+1$ in $1 /\left(z-\lambda_{k}\right)$ without constant term. This representation yields the bound

$$
\begin{aligned}
\left|\Psi^{(r)}\left(\lambda_{k}\right)\right| & \exp \left(q\left|\operatorname{Re} \lambda_{k}\right|\right) \\
& \leqq \frac{1}{2 \pi}\left(\int_{\Gamma}\left|\Psi\left(x+i y_{\Gamma}\right)\right|^{2} e^{2 q|x|} d x\right)^{1 / 2}\left(\int_{\Gamma}\left|P_{r q}\left(\frac{1}{x+i y_{\Gamma}-\lambda_{k}}\right)\right|^{2} d x\right)^{1 / 2} .
\end{aligned}
$$

Let

$$
W_{q r k}^{-1}=\left(\int_{\Gamma}\left|P_{r q}\left(\frac{1}{x+i y_{\Gamma}-\lambda_{k}}\right)\right|^{2} d x\right)^{1 / 2} .
$$

It is natural to introduce in the space $S$ a Fréchet topology given by the norms

$$
\|\sigma\|_{q}=\sup _{r, k}\left|\sigma_{r k}\right| W_{q r k} \exp \left(q\left|\operatorname{Re} \lambda_{k}\right|\right), \quad q=1,2, \ldots
$$

The inequality (15) clearly implies

$$
\|\rho(\Psi)\|_{q} \leqq(1 / 2 \pi)\|\Psi\|_{2 q, n},
$$

where $n$ is the index associated with $\Lambda$ (cf. (5) and the definition of the contour $\Gamma$, which depends on $n$ ). Therefore we have established

LeMmA 5. The mapping $\rho: \mathscr{F} \mathscr{M} \rightarrow S$ is continuous.

In view of (16), the dual space $S^{\prime}$ of $S$ consists of all numerical sequences

$$
t=\left\{t_{r k}\right\}, \quad r=0, \ldots, p_{k}-1, \quad k=1,2, \ldots,
$$

such that there exists some $q<\infty$ for which

$$
\sum_{k, r}\left|t_{r k}\right| W_{q r k}^{-1} \exp \left(-q\left|\operatorname{Re} \lambda_{k}\right|\right)<\infty
$$

We have

$$
\langle\sigma, t\rangle=\sum_{r, k} \sigma_{r k} t_{r k}
$$

Lemma 6. The quotient space $\mathscr{F} \mathscr{M} / \operatorname{ker} \rho$ is isomorphic as a Fréchet space to the sequence space $S$ under the mapping induced by $\rho$.

Proof. The mapping $\rho$ automatically induces an injective mapping of the quotient space $\mathscr{F} \mathscr{M} /$ ker $\rho$ into $S$. We shall show that this is actually surjective. 
Once this is done, the open mapping theorem (cf. $[4$, p. 75]) implies the stated isomorphism.

Proving surjectivity amounts to solving a universal interpolation problem, which we now proceed to do. Thus let $t=\left\{t_{r k}\right\}$ be any given sequence of the space $S$. Consider first the interpolation problem of finding a function $V_{k} \in \mathscr{F} \mathscr{M}$ such that $V_{k}$ vanishes up to order $p_{v}$ at $\lambda_{v}$ for all indices $\nu$ distinct from $k$ and such that $V_{k}^{(r)}\left(\lambda_{k}\right)=t_{r k}, r=0, \ldots, p_{k}-1$ for the index $k$.

Let $b_{k}$ denote the Blaschke product relative to the strip $A$ with zeros at $\lambda_{v}$ of order $p_{v}$ for all $\nu \neq k$, precisely. Since the $\lambda_{v}$ are zeros of $M_{0} \in \mathscr{F} \mathscr{M}, b_{k}$ certainly exists. There exist numbers $s_{0}, s_{1}, \ldots, s_{p}\left(p=p_{k}-1\right)$ such that the function

$$
f_{k}(\lambda) \equiv b_{k}(\lambda)\left(s_{0}+s_{1}\left(\lambda-\lambda_{k}\right)+\cdots+\left(s_{p} / p !\right)\left(\lambda-\lambda_{k}\right)^{p}\right)
$$

satisfies the interpolation conditions

$$
f_{k}^{(r)}\left(\lambda_{k}\right)=t_{r k}, \quad r=0, \ldots, p ;
$$

i.e.

$$
\sum_{v=0}^{r}\left(\begin{array}{l}
r \\
v
\end{array}\right) b_{k}^{(r-v)}\left(\lambda_{k}\right) s_{v}=t_{r k}
$$

Since $b_{k}\left(\lambda_{k}\right) \neq 0$, this triangular system has a unique solution $s_{0}, \ldots, s_{p}$.

Define

$$
V_{k}(\lambda)=f_{k}(\lambda) \exp \left(-N_{k}\left(\lambda-\lambda_{k}\right)^{2 p_{k}}\right) .
$$

Then $V_{k}$ has the same interpolating properties (18) as $f_{k}$ and belongs to $\mathscr{F} \mathscr{M}$ for each $N_{k}>0$. It only remains to check that by choosing $N_{k}$ large enough we can have

$$
V=\sum_{k} V_{k} \in \mathscr{F} \mathscr{M}
$$

i.e. $\|V\|_{q n}<\infty, \forall q, n$. First, take one sequence of positive constants $\varepsilon_{k}$ such that

$$
\sum_{k} \varepsilon_{k} W_{q o k}^{-1}<\infty, \quad \forall q\left({ }^{3}\right) .
$$

(Actually, $W_{\text {qok }}$ does not depend upon $q$ !) By going over to an obvious upper bound, everything comes down to showing that the sum

$$
\sum_{k} \sup _{|y| \leq a}\left(\int_{-\infty}^{\infty}\left|f_{k}(x+i y)\right|^{2} \exp \left(-2 N_{k} \operatorname{Re}\left(x+i y-\lambda_{k}\right)^{2 p_{k}}\right) e^{2 q|x|} d x\right)^{1 / 2}
$$

is finite for each $q<\infty$. To this end, divide the range of integration into $A_{k} \equiv\left\{\left|\operatorname{Re}\left(z-\lambda_{k}\right)\right| \leqq \theta_{k}\right\}$ and $B_{k} \equiv\left\{\left|\operatorname{Re}\left(z-\lambda_{k}\right)\right| \geqq \theta_{k}\right\}$ and choose $\theta_{k}>0$ so that

$$
\left|\int_{A_{k}}\right| \leqq \text { Const. }\left|t_{o k}\right|^{2} \exp \left(2 q\left|\operatorname{Re} \lambda_{k}\right|\right) \varepsilon_{k}^{2}
$$

(3) $W_{\text {qok }}$ denotes $W_{\text {qrk }}$ with $r=0$. 
for a constant independent of $k$ and for all $N_{k}$. We use here the interpolatory property of $f_{k}$ for $r=0$. This upper bound is

Const. $\left(\left|t_{o k}\right| W_{q o k} \exp \left(q\left|\operatorname{Re} \lambda_{k}\right|\right)\right)^{2} \cdot \varepsilon_{k}^{2} / W_{q o k}^{2} \leqq$ Const. $\|t\|_{q}^{2} \cdot \varepsilon_{k}^{2} / W_{q o k}^{2}$.

Fixing such $\theta_{k}$, we have

$$
\begin{array}{r}
\left|\int_{B_{k}}\right| \leqq \sup _{\operatorname{Re}}\left|f_{k}(z)\right|^{2} \exp \left(-N_{k} \operatorname{Re}\left(z-\lambda_{k}\right)^{\left.2 p_{k}\right)}\right. \\
\cdot \int_{B_{k}} \exp \left(q|x|-N_{k} \operatorname{Re}\left(z-\lambda_{k}\right)^{2 p_{k}}\right) d x
\end{array}
$$

a bound which is less than

$$
\frac{1}{k^{4}} \int_{B_{k}} \exp \left(q|x|-N_{k} \operatorname{Re}\left(z-\lambda_{k}\right)^{\left.2 p_{k}\right)} d x\right.
$$

for all sufficiently large $N_{k}$. We now fix $N_{k}$ large enough so that in addition

$$
\int_{B_{k}} \exp \left(k|x|-N_{k} \operatorname{Re}\left(z-\lambda_{k}\right)^{\left.2 p_{k}\right)} d x \leqq 1 .\right.
$$

Now consider any index of $q$ whatsoever. For indices $k>q$, the upper bound appearing in (23) is less than $1 / k^{4}$. Therefore the sum in (21) over indices $>q$ is at most

$$
\sum_{k>q}\left(\text { Const. }\|t\|_{q}^{2} \cdot \frac{\varepsilon_{k}^{2}}{W_{q o k}^{2}}+\frac{1}{k^{4}}\right)^{1 / 2} \leqq \text { Const. } \sum_{k>q}\left(\|t\|_{q} \cdot \frac{\varepsilon_{k}}{W_{q o k}}+\frac{1}{k^{2}}\right)<\infty,
$$

which proves that $V \in \mathscr{F} \mathscr{M}$, since (19) defines a function holomorphic in $|y|<a$. The interpolating properties of the $V_{k}$ combine to give

$$
V^{(r)}\left(\lambda_{k}\right)=t_{r k}, \quad \forall r, k
$$

The proof of Lemma 6 is complete.

Lemma 6 allows us to regard $\Lambda$ as an element of the dual space $S^{\prime}$. Thus, in view of (17),

$$
\langle\Lambda, \Psi\rangle=\sum_{k, r} \Lambda_{k r} \Psi^{(r)}\left(\lambda_{k}\right), \quad \forall \Psi \in \mathscr{F} \mathscr{M},
$$

where the numerical sequence $\left\{\Lambda_{k r}\right\}$ satisfies

$$
\sum_{k, r}\left|\Lambda_{k r}\right| \exp \left(-m\left|\operatorname{Re} \lambda_{k}\right|\right) W_{m r k}^{-1}<\infty, \quad \exists m<\infty .
$$

(24) means that $\Lambda$ is a sum of derivatives of Dirac measures at the points $\lambda_{k}$. Therefore, after a Fourier inversion, we obtain at once the weakly convergent series in $\mathscr{M}^{\prime}$ :

$$
\lambda=\mathscr{F}^{-1} \Lambda=\sum_{k, r} \Lambda_{k r}(i x)^{r} \exp \left(i \lambda_{k} x\right)
$$

This completes the proof of the first part of the theorem. 
The converse part is trivial. For suppose that sequences $\left\{\lambda_{k}\right\},\left\{p_{k}\right\},\left\{c_{r k}\right\}$ appearing in a weakly convergent expansion (2) of an element $\lambda \in \mathscr{M}^{\prime}$ are given, where $\left\{c_{r k}\right\} \in S^{\prime}$ and $\left\{\lambda_{k}\right\},\left\{p_{k}\right\}$ are the zeros with multiplicities of some function $M_{0} \in \mathscr{F} \mathscr{M}$. Put

$$
\langle\Lambda, \sigma\rangle=\sum_{k, r} c_{r k} \sigma_{r k}, \quad \sigma \in S .
$$

The proof of Lemma 6 remains valid, so we can pull back $\Lambda$ to a continuous linear form on $\mathscr{F} \mathscr{M}$ acting according to the formula

$$
\langle\Lambda, \Psi\rangle=\sum_{k, r} c_{r k} \Psi^{(r)}\left(\lambda_{k}\right)
$$

In particular, for the function $M_{0} \in \mathscr{F} \mathscr{M}$ which vanishes as stated, we have $\left\langle\Lambda, M_{0}\right\rangle=0$, as well as

$$
\left\langle\Lambda, M_{0} e^{i \alpha x}\right\rangle=0, \quad \forall \alpha \in R .
$$

Going over to $\lambda=\mathscr{F}^{-1} \Lambda$, this means that $\lambda$ is orthogonal to all translates of $m_{0}$ $=\mathscr{F}^{-1} M_{0}$; i.e. $\lambda$ is mean-periodic in $\mathscr{M}^{\prime}$.

\section{REFERENCES}

1. E. J. Akutowicz, Sur la moyenne-périodicité. I, J. Math. Pures Appl. (9) 48 (1969), 307344. MR 41 \#814.

2. G. Köthe, Dualität in der Funktionentheorie, J. Reine Angew. Math. 191 (1953), 30-49. MR 15, 132.

3. V. P. Palamodov, Linear differential operators with constant coefficients, "Nauka", Moscow, 1967 (Russian). MR 39 \#4517.

4. K. Yosida, Functional analysis, Die Grundlehren der math. Wissenschaften, Band 123, Academic Press, New York; Springer-Verlag, Berlin, 1965. MR 31 \#5054.

5. L. Ehrenpreis, Fourier analysis in several complex variables, Interscience Monographs in Pure and Appl. Math., vol. 27, Interscience, New York, 1970.

FACUlté des SCIENCES, MontPellier, France

INSTITUTE FOR ADVANCED STUdy, Princeton, New Jersey 08540 